\title{
Wnt/ $\beta$-catenin Signaling Pathway Promotes Transdifferentiation from Fetal Skin Fibroblasts to Keratinocyte-like Cells
}

Hongqing Zhao

JinZhou Medical College: Jinzhou Medical University

Xinzhu Liu

Fourth Medical Center of PLA General Hospital

Jiachen Sun

Fourth Medical Center of PLA General Hospital

Yuezeng Niu

Fourth Medical Center of PLA General Hospital

Kun Zhang

Jinzhou Medical University

Zhisheng Li

Jinzhou Medical University

Jinglong Ma

Fourth Medical Center of PLA General Hospital

Jianqiu Yang

Jinzhou Medical University

chuanan Shen ( $\square$ shenchuanan@126.com )

Fourth Medical Center of PLA General Hospital https://orcid.org/0000-0001-8298-8355

\section{Research Article}

Keywords: Cell transdifferentiation, Fetal skin fibroblast, Keratinocytes, Keratinocyte-like cells, Mesenchymal-epithelial transformation(MET), Epithelial-mesenchymal transformation(EMT), Wound healing

Posted Date: November 16th, 2021

DOI: https://doi.org/10.21203/rs.3.rs-1068381/v1

License: (c) (i) This work is licensed under a Creative Commons Attribution 4.0 International License. Read Full License 


\section{Abstract}

Background: Wound healing is a dynamic, sequential,and complex physiological process, including a variety of cellular events, such as proliferation, adhesion, chemotaxis, and apoptosis. Skin fibroblasts and keratinocytes are the two most important cells involved in wound repair, and Relying on the proliferation and differentiation of keratinocytes to form epithelium to completely cover the wound is the most ideal result for wound repair, so expanding the source of keratinocytes is a huge challenge. In this study, we examined the phenomenon that fetal skin fibroblasts spontaneously transdifferentiated into keratinocytelike cells in conventional culture, and evaluated the characteristics of KLCs and the potential mechanisms of the transdifferentiation process.

Methods: HFF-1 were routinely cultured in ordinary DMEM medium for more than 40 days,and observed the cell morphology. The cytological properties of KLCs at the cellular and molecular levels were detected by RT-PCR, Western-blot, immunofluorescence, Transwell, and cell scratch experiments. The functionality and safety of KLCs were determined through wound healing and tumorigenicity experiments. And highthroughput transcriptome sequencing (RNA-seq) was performed to explore the mechanism underlying HFF-1 transdifferentiation.

Results: The transdifferentiation process started on the 25th day and was completed by the 40th day. KLCs and KCs had similar expressions at the molecular and protein levels, both functioned similarly in wound healing and were non-tumorigenic.RNA-seq revealed that the transdifferentiation process was regulated by the activation of the classical Wnt/ $\beta$-catenin signaling pathway, which could shorten the process to 10 days.

Conclusion: This study demonstrates that HFF-1 can spontaneously transdifferentiate into KLCs with conventional culture conditions, and the $\mathrm{Wnt} / \beta$-catenin signaling pathway regulates the transdifferentiation process.

\section{Background}

Cell transdifferentiation refers to the process by which one type of differentiated cell is transformed into another type of differentiated cell in terms of structure and function. Yamanaka $\mathrm{K}$ et al discovered for the first time that a combination of four transcription factors (Oct4, Sox2, Klf4 and c-myc), transfected with a viral vector, could dedifferentiate terminally differentiated somatic cells into a cell type similar to embryonic stem cells (ESCs), induced pluripotent stem cells (iPSCs) [1], which overturned the previous knowledge that the developmental path of terminally differentiated cells was irreversible.

Transdifferentiation between different types of cells has also been reported through further research. For example, Kocaefe $\mathrm{YC}$ et al transdifferentiated rat adipocytes into myoblasts [2]. leda $\mathrm{M}$ et al transdifferentiated fibroblasts from mouse heart and tail tip into cardiomyocytes [3]. Vierbuchen $\mathrm{T}$ et al transdifferentiated fibroblasts into neurocytes [4]. Ambasudhan $\mathrm{R}$ et al transdifferentiated neonatal and adult skin fibroblasts into functional neurocytes [5]. Szabo $E$ et al transdifferentiated human skin 
fibroblasts into hematopoietic progenitor cells [6], and Sekiya S et al transdifferentiated mouse fibroblasts into liver-like cells [7].

The repair of wounds depends on the division and proliferation of keratinocytes, which fuses gradually and reform the epidermis. When extensive burns occur, the structure and function of the skin and its accessory organs are severely damaged. The current therapeutics in clinical practice mainly rely on autologous or allogeneic skin grafting. However, for patients with extensive burns, the available skin donor sites are limited, which hardly meet the needs of large-area skin grafting and thus limit the patient's chances of survival. Keratinocytes (KCs) are the target cells for wound repair, and it remains a huge challenge to obtain keratinocytes on a large scale. Studies have found that KCs can be derived by transdifferentiation from embryonic stem cells (ESCs), iPSCs, adipose stem cells (ATSCs), wound resident mesenchymal cells and fibroblasts [8]. Bilousova et al found that Oct4, Sox2, c-Myc and Klf4 genes could be transfected with viral vectors to dedifferentiate fibroblasts into iPSCs, and then by incubation with a medium containing retinoic acid (RA) and a medium containing Bmp4, the induced pluripotent stem cells were further differentiated into keratinocytes. Chen et al. reported that two genes, P63 and KLF4, were transfected to directly transdifferentiate fibroblasts into keratinocytes [9]. Cell reprogramming provides a new idea for the source of KCs. Although gene transfection using viral vectors can induce cell transdifferentiation, this method is difficult to use in clinical practice due to toxicity, tumorigenic risk, and especially low transdifferentiation efficiency.The purpose of wound healing is to quickly restore the structure and function of the skin, but in extreme cases this will lead to scar formation. Scar tissue is a dysfunctional fibrous tissue [10]. Unlike wound healing in adults, perfect tissue regeneration is observed in the fetus, which is characterized by the absence of scars [11].

When we routinely cultured fetal skin fibroblasts, a wonderful phenomenon of cell transdifferentiation was observed, that is, HFF-1 spontaneously transdifferentiated into keratinocyte-like cells (KLCs) in a regular high-sugar DMEM medium in a time-accumulating manner, and the transformed KLCs showed similar behaviors and effects to KCs in terms of cell characteristics, cell behavior and wound healing, and were not tumorigenic.To further explore the potential mechanism of HFF-1 transdifferentiating into KLCs, we performed RNA-seq. Through analysis, we found that this time-dependent HFF-1 transformation is regulated by the $\mathrm{Wnt} / \beta$-catenin signaling pathway. The discovery of cell transdifferentiation has opened up a new horizon for skin regeneration and scarless wound healing in severe burns.

\section{Methods}

\section{Cell isolation and culture}

Human skin tissue specimens were taken from our burn center, with ethical approval and informed consent. Human skin KCs were extracted as previously described [12]. In brief, the obtained skin tissue was cleaned with PBS (500ml,Cat\#G4202-500ML, Servicebio) for three times before the subcutaneous tissue and adipose tissue was removed, and the skin tissue was trimmed to make it regular. Subsequently, the skin tissue was placed into Dispase II enzyme (2.5mg/mL,Cat\#D4693-1G,Sigma) for 
digestion overnight at $4^{\circ} \mathrm{C}$, and the epidermis and dermis were separated the next day.

Then,trypsin/ethylenediaminetetraacetic acid $\left(0.25 \%\right.$,Cat\#25200072,Gibco) preheated at $37^{\circ} \mathrm{C}$ was used to grind and digest the skin tissue for 5 minutes, and then the cell suspension was sucked. The said trypsinization was repeated three times until the skin tissue was completely digested. The cell suspension was filtered through a 70um cell sieve and sterilized (0.22um,Cat\#SLGP033RB,Millex), centrifuged at 13000 rpm for 3 minutes, and washed once with PBS. Afterwards, the cells were seeded on a 6-well plate (Cat\#3516S,Corning), pre-coated with type IV collagen (Cat\#C6745,Merck), and routinely cultured with 1\% HKGS (Cat\#S0015,Gibco) using epilife medium (Cat\#Mepicf500,Gibco). Fetal skin fibroblast cell line (HFF-1,The cell identification certificate is in the supplementary material 1) was purchased from JiKai Gene (Shanghai,China) and grown in DMEM high glucose medium (Cat\#SH30022.01,Hyclone) with 10\% FBS (Cat\#10099141C,Gibco). The third-generation cells were used in the experiment. HepG2 liver cancer cells were donated by Peking University People's Hospital and cultured under the same conditions as described above. All cells were cultured in a $5 \% \mathrm{CO}_{2}, 37^{\circ} \mathrm{C}$ incubator, and the medium was changed every three days. All cells were grown without antibiotics.

\section{Flow Cytometry}

Flow cytometry was used to quantitatively analyze HFF-1 vimentin and CK14 positive cells in the process of transdifferentiation. Simply put, the cell suspension at each time point in the HFF-1 culture process was taken fixed with $4 \%$ paraformaldehyde fixative (Cat\#P0099,Beyotime) at $4^{\circ} \mathrm{C}$ for $30 \mathrm{~min}$, and then the permeation buffer TritonX-100 (Cat\#P0096,Beyotime) was used to infiltrate the cells and further incubated with the corresponding antibody , rabbit anti-CK14 (Cat\#ab192056,Abcam) and mouse antiVimentin (Cat\#ab8069,Abcam)overnight at $4^{\circ} \mathrm{C}$. After incubation, the cells were washed to form a $200 \mu \mathrm{l}$ cell suspension. Flow cytometry analysis of cell suspension was performed with FACScan (Beamcyte1026,China).

\section{RT-PCR analysis}

RNA extraction and qRT-PCR detection were performed as previously described [13]. The total RNA was extracted with Trizol (Cat\#15596026,Thermo Fisher), and the precipitate was washed with $75 \%$ ethanol and dissolved in distilled water without ribonuclease. The purity and concentration of total RNA were determined with an ultraviolet spectrophotometer (NanoDrop 2000,Thermo Fisher,Waltham, MA,USA). As

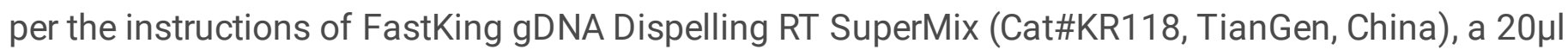
reverse transcription reaction system was prepared and incubated at $42^{\circ} \mathrm{C}$ for 15 min and $95^{\circ} \mathrm{C}$ for 3 min in a T100 thermal cycler (BIO-RAD, Hercules, CA, USA), and then stored at $4^{\circ} \mathrm{C}$. Afterwards, Top Green qPCR SuperMix, Nuclease-free Water, cDNA template, and forward and reverse ribonucleic acid primers were added to form a $20 \mu \mathrm{l}$ reaction system. The amplification was performed in the CFX Connect realtime system (BIO-RAD,Hercules,CA,USA) as follows: $94^{\circ} \mathrm{C}$ for $30 \mathrm{~s}, 95^{\circ} \mathrm{C}$ for $5 \mathrm{~s}, 56^{\circ} \mathrm{C}$ for $15 \mathrm{~s}, 72^{\circ} \mathrm{C}$ for 10 $s$ for 45 cycles. The CT value of each well was detected and recorded, and $2-\Delta \Delta C t$ was used to normalize 
the gene expression in the sample to the expression of GAPDH. The primer sequence was shown in Table 1.

\section{Western blot analysis}

The cell pellets of each group were collected, washed with PBS for three times, and then the total protein was extracted. In short, protease inhibitor (Cat\#36978, Thermo Fisher) and phosphatase inhibitor (Cat\#A32957,Thermo Fisher) were added in RIPA lysate (Cat\#89901,Thermo Fisher) with a volume ratio of 1:1:100, and homogenized on ice.The protein concentration was determined with an ultraviolet spectrophotometer, and the target protein was detected by Western blot. $40 \mu \mathrm{g}$ of total protein was taken for sodium dodecyl sulfate-polyacrylamide gel electrophoresis and wet transferred to PVDF membrane (Cat\#IPVH00010,Millipore,Merck), and the sample was blocked with 5\% skim milk at room temperature for $1 \mathrm{~h}$. Then the sample was incubated respectively with anti-CK14 (Cat\#60320-1-AP,1:1000,mouse monoclonal,Proteintech),anti-CK5(Cat\#66727-1-Ig,1:1000,mouse,monoclonal,Proteintech),Integrin Beta 1(Cat\#66315-1-Ig, 1:1000, rabbit monoclonal, Proteintech), anti-Ecadherin(Cat\#ab1416,1:1000,mousemonoclonal,Abcam),antiCK19(Cat\#ab76539,1:1000,rabbitmonoclonal,Abcam),anti- $\beta$ catenin(Cat\#8480S,1:1000,rabbitmonoclonal,CST), antiVimentin(Cat\#5741S,1:1000,rabbit,monoclonal,CST), anti-P-ß-catenin(Cat\#5741S, 1:1000, rabbit monoclonal, CST), anti-GSK-3 $\beta$ (Cat\#5741S, 1:1000, rabbit monoclonal,CST), anti-P-GSK-3 $\beta$ (Cat\#5558S, 1:1000, rabbit monoclonal,CST), anti-Axin1(Cat\#2087S,1:1000,rabbit,monoclonal,CST),anti-

LEF1(Cat\#2230S,1:1000,rabbit,monoclonal,CST), anti-

ZEB1(Cat\#3396S,1:1000,rabbit,monoclonal,CST),anti-GAPDH(Cat\#5174S,1:2000, rabbit monoclonal,CST) overnight at $4^{\circ} \mathrm{C}$. The next day after rewarming to room temperature, horseradish peroxidase-labeled anti-rabbit IgG (Cat\#7074S,1:5000,CST) and anti-mouse IgG (Cat\#7076S,1:5000,CST) were added correspondingly and incubated at room temperature for 1 hour. After chemiluminescence and image development, the image was exposed using a gel imaging system (Bio-RAD, Hercules, CA, USA).

\section{CCK8 assay}

After the cells were trypsinized (Cat\#KR11825200056, Gibco), HFF-1, KLCs and KCs were seeded in a 96well plate, each group of cells with 6 wells. After they grew adherently for 8h. 10ul CCK8 reagent (Cat\#HY$\mathrm{K} 0301, \mathrm{MCE}$ ) was added to each well, and the cells were incubated at $37^{\circ} \mathrm{C}$ for $4 \mathrm{~h}$ before a multifunctional microplate reader (Biotek Synergy2, USA) was employed to measure the absorbance 12, 24,36 , 48hour at $450 \mathrm{~nm}$ wavelength.

\section{Cell migration analysis}


Scratch assay: After the cells were trypsinized, HFF-1, KLCs and KCs were seeded in a 6-well plate at a density of $5 \times 10^{5}$ cells/well. After overnight incubation, a $200 \mu$ l pipette tip (Cat\#TF200-RS, Axygen) was used to form wound scratches. Each well was cleaned with PBS for three times to remove sloughed cells. At $0,12,24$, and 48 hour, images were taken with a Leica optical microscope to calculate the number of migrated cells.

Transwell assay: After the cells were digested, Transwell assay was performed in a 24-well Transwell chamber (Cat\#3422, Corning). In brief, the mixed cell culture medium (DMEM high glucose medium + $10 \%$ fetal calf serum $+1 \%$ EGF) was added to the lower chamber, and $200 \mu$ l of DMEM high glucose medium was added to the upper chamber after resuspending the cells. After incubation at $37^{\circ} \mathrm{C}$ for $24 \mathrm{~h}$, the cells were taken out of the chamber, and the cells that migrated to the bottom of the chamber were stained with crystal violet (Cat\#C8470, Solarbio, China), and each well was counted manually under a microscope.

\section{Immunofluorescence}

The immunofluorescence test was carried as previously described [14]. Simply put, cells growing on a glass slide were fixed with $4 \%$ paraformaldehyde for $15 \mathrm{~min}$, and $0.25 \%$ Triton X-100 (Cat\#X100-100ml, Sigma) diluted in PBS for 10 min was used for infiltration at room temperature.To block non-specific epitope binding, cells were incubated with phosphate buffer containing $1 \%$ bovine serum albumin (Cat\#A1933, Merck) and 0.1\% Tween-20 (Cat\#P7949,Sigma) for $1 \mathrm{~h}$, and then incubated overnight at $4^{\circ} \mathrm{C}$ with the following primary antibodies: mouse anti-CK14, rabbit anti-Vimentin, and anti-a-SMA (Cat\#23081-1-AP, mouse monoclonal, Proteintech). After that, the cells were incubated with donkey antimouse or anti-rabbit secondary antibodies conjugated with Alexa Fluor 488 (Cat\#ab150077,Abcam) or Alexa Fluor 568 (Cat\#ab175704, Abcam), and the nuclei were reversely stained with DAPI (Cat\#d1306, Thermo Fisher).

\section{Wound healing model and treatment}

All animal experiments were approved by the appropriate ethics committee of our hospital. Eight-week-old male BALB/c mice (SPF Biotechnology Beijing, China) were randomly divided into four groups, each with 15 mice. As described by Wu et al [15], a full-thickness skin defect of $5 \mathrm{~mm} \times 5 \mathrm{~mm}$ was created on mice's dorsum. The treatment plan was: (1) PBS group: 100ul PBS was administered into the wound surface; (2) HFF-1 group: $100 \mathrm{ul} \mathrm{HFF-1} \mathrm{(} 1 \times 10^{7}$ cells/ml) cell suspension was administered into the wound surface; (3) KLCs group: $100 \mathrm{ul} \mathrm{KLCs}\left(1 \times 10^{7}\right.$ cells $\left./ \mathrm{ml}\right)$ cell suspension was administered into the wound; (4) KCs group: $100 \mathrm{ul} \mathrm{KCs}\left(1 \times 10^{7}\right.$ cells $\left./ \mathrm{ml}\right)$ cell suspension was administered to the wound. The above treatment plan was repeated every day, and digital photos were taken on the $0^{\text {th }}, 3^{\text {rd }}, 6^{\text {th }}$, and $9^{\text {th }}$ days. Wound healing rate $=$ (original wound area-actual wound area) $/$ original wound area $\times 100 \%$. 


\section{Tumorigenicity analysis}

Eight-week-old male BALB/c-Nu nude mice (SPF Biotechnology Beijing, China) were randomly divided into three groups: KLCs, KCs and Hepg2, with 5 mice randomly selected from each group. Cells from KLCs, KCs and Hepg2 groups were suspended respectively in PBS $\left(5 \times 10^{6}\right.$ cells per $\left.100 \mu l\right)$. We injected $100 \mu \mathrm{l}$ of this cell suspension $\left(5 \times 10^{6}\right.$ cells) into the subcutaneous tissue of nude mice. The mice were sacrificed 40 days after cell transplantation. The tumor size was measured and the tumor was dissected for histopathological examination [16].

\section{HE staining}

In brief, fresh tissues were fixed in $4 \%$ paraformaldehyde for $24 \mathrm{~h}$, dehydrated, paraffin embedded, sectioned, and H\&E stained as previously described [17]. The prepared paraffin sections were deparaffinized, stained with hematoxylin-eosin (Cat\# C0105M, Beyotime), and finally dehydrated and mounted. The histopathological changes were observed with an optical microscope (Nikon DS-U3).

\section{Immunohistochemical analysis}

Tissue sections were taken, deparaffinized with xylene, dehydrated in a decreasing ethanol gradient, and incubated with citric acid buffer. The sample was incubated overnight with mouse anti-human CK5 at $4^{\circ} \mathrm{C}$, washed with PBS for three times, and then incubated with secondary antibody for corresponding specimens at room temperature for 1 hour. Subsequently, the sample was incubated with diaminobenzidine and sectioned, and re-stained with hematoxylin to obtain images.

\section{RNA-seq analysis}

Total RNA was extracted using Trizol reagent kit (Invitrogen, Carlsbad, CA, USA) according to the manufacturer's protocol. RNA quality was assessed on an Agilent 2100 Bioanalyzer (Agilent Technologies, Palo Alto, CA, USA) and checked using RNase free agarose gel electrophoresis. After total RNA was extracted, eukaryotic mRNA was enriched by Oligo(dT) beads, while prokaryotic mRNA was enriched by removing rRNA by Ribo-Zero TM Magnetic Kit (Epicentre, Madison, WI, USA). Then the enriched mRNA was fragmented into short fragments using fragmentation buffer and reverse transcripted into CDNA with random primers. Second-strand CDNA were synthesized by DNA polymerase I, RNase $\mathrm{H}, \mathrm{dNTP}$ and buffer. Then the cDNA fragments were purified with QiaQuick PCR extraction kit (Qiagen, Venlo, The Netherlands), end repaired, A base added, and ligated to Illumina sequencing adapters. The ligation products were size selected by agarose gel electrophoresis, PCR amplified, and sequenced using Illumina Novaseq6000 by Gene Denovo Biotechnology Co. (Guangzhou, China). DNA/RNA/Small RNA/cDNA library sequencing was performed on the lllumina HiseqTM 2500/4000 by Gene Denovo Biotechnology Co., Ltd (Guangzhou, China). 


\section{DEG analysis}

RNAs differential expression analysis was performed by DESeq2 software between two different groups and by edgeR between two samples $[18,19]$. The genes/transcripts with the parameter of false discovery rate (FDR) below 0.05 and absolute fold change $\geq 2$ were considered differentially expressed genes/transcripts.

\section{Principal component and pathway enrichment analysis}

Principal component analysis (PCA): PCA was performed with R package gmodels (http://www.rproject.org/) in this experience. PCA is a statistical procedure that converts hundreds of thousands of correlated variables (gene expression) into a set of values of linearly uncorrelated variables called principal components. PCA is largely used to reveal the structure/relationship of the samples/data.

Pathway analysis: Genes usually interact with each other to play roles in certain biological functions. Pathway-based analysis helps to further understand genes biological functions. KEGG is the major public pathway-related database. Pathway enrichment analysis identified significantly enriched metabolic pathways or signal transduction pathways in DEGs comparing with the whole genome background [20]. The calculated p-value was gone through FDR Correction, taking FDR $\leq 0.05$ as a threshold. Pathways meeting this condition were defined as significantly enriched pathways in DEGs.

\section{Statistical analysis}

All data were expressed as mean \pm standard deviation (SD). The $t$ test was used for the analysis between two groups, and the one-way analysis of variance (ANOVA) was used to compare the data of three or more groups. $P$ values $<0.05$ were considered statistically significant. Statistical analysis was performed with the software GraphPad Prism 5.

\section{Results}

\section{HFF-1 transforms into KLC spontaneously in a time- accumulated manner}

When HFF-1 was grown in conventional culture medium for more than 40 days without any special inducers, we unexpectedly found that it could spontaneously transdifferentiate into KLCs. The entire transdifferentiation process started on the 25th day, marked with the "diamond-like" appearance of the cell colony, and all HFF-1 appeared "diamond-like" by the 40th day (Figure 1a, 1b). To quantify the cell types in the process of transdifferentiation, CK14-positive cells and vimentin-positive cells were evaluated by flow cytometry. The percentage of CK14-positive cells was $19.55 \pm 0.43 \%, 49.92 \pm 0.38 \%, 76.63 \pm 0.64 \%$, 
and $98.10 \pm 0.66 \%$ on the 10 th, 20th, 30th and 40th day, respectively; the percentage of vimentin-positive cells on the 10th, 20th, 30th and 40th days were72.42 $\pm 0.48 \%, 59.19 \pm 0.10 \%, 22.41 \pm 0.33 \%$, and $3.54 \pm 0.26 \%$ (Figure 1c). Immunofluorescence showed that HFF-1 transformed into KLCs in a time-accumulated manner. The number of positive cells for epidermal cell-specific protein CK14 gradually increased [21], while the number of positive cells of fibroblast-specific proteins Vimentin and a-SMA gradually decreased with time [22,23]. By the 40th day, the number of positive cells of vimentin or a-sma were significantly reduced and could hardly be detected (Figure 1d,1e).In short, HFF-1 transdifferentiated to KLCs gradually with time, with decreasing HFF-1 and increasing KLCS, and a entire transformation occurred on the 40th day.

\section{Characteristics of KLCs}

To explore the characteristics of KLCs after transdifferentiation, we compared KLCs with HFF-1 and KCs. From a molecular level, the Western blot results revealed that the epithelial marker protein Ecadherin [24] as well as KCs-labeled proteins, including CK5 [25], CK14 [26], CK19 [27,28] and Integrin beta1 [29] were expressed in KCs and KLCs, but not in HFF-1. Meanwhile, the HFF-1 marker protein Vimentin [22,23] was expressed in HFF-1, but not in KLCs or KCs (Figure 2a). Consistent with the Western blot results, qRT-PCR results confirmed that although the mRNA expression levels of E-cadherin and CK19 in KLCs were lower than those in KCs, they were 40 times and 20 times that in HFF-1, respectively. Interestingly, the mRNA expression level of CK14 in KLCs was 100 times that in KCs. The expression of CK14 mRNA in KLCs and KCs was much higher than that in HFF-1. In the meantime, the mRNA expression of Vimentin species in HFF-1 was higher than that in KLCs and KCs (Figure 2b).

From the cellular level, CCK8 assay showed that the proliferation rate of KCs and KLCs was significantly higher than that of HFF-1 at 24h, 48h and $72 \mathrm{~h}$ after the adherent growth of the cells, and the proliferation group of KLCs was higher than that of KCs at each time point (Figure 2c). In terms of cell migration rate, according to the scratch assay results, there was no significant difference between HFF-1, KLCs and KCs $12 \mathrm{~h}$ after the establishment of the cell wound model. However, at $24 \mathrm{~h}$ and $48 \mathrm{~h}$, the migration rate of HFF-1 was significantly higher than that of KLCs and KCs (Figure 2e). The Transwell assay further showed that the number of migrating cells decreased in HFF-1, KLCs and KCs (Figure 2d). These results indicated that the proliferative ability of KCs and KLCs was higher than that of HFF-1, but the migration efficiency of HFF-1 was significantly higher than that of KCs and KLCs.

\section{KLCs accelerate wound healing}

To explore the therapeutic effect of KLCs on wound healing after transdifferentiation, PBS, HFF-1, KLCs and KCs suspension were administered into to a full-thickness skin defect wound $(5 \mathrm{~mm})$ on the back of the mice, and the wound was inspected on the 3rd, 6th, and 9th days (Figure 3a). In the PBS group, the wound healing rates on the $3 \mathrm{rd}$, 6th and 9 th days were $51.80 \pm 0.67 \%, 73.80 \% \pm 0.32$ and $90.20 \pm 4.52 \%$, respectively; in the HFF-1 group, the wound healing rates on the $3 \mathrm{rd}$, 6th and 9 th days were $60.60 \pm 0.53 \%$, 
$85.40 \pm 0.69 \%$ and $92.20 \pm 3.97 \%$, respectively; in the KLCs group, the wound healing rates on the 3rd, 6 th and 9th days were $72.60 \pm 4.63 \%, 92.20 \pm 6.02 \%$ and $97.40 \pm 3.18 \%$, respectively; in the KCs group, the wound healing rates on the $3 \mathrm{rd}$, 6 th and 9 th days were $82.80 \pm 2.81 \%, 89.00 \pm 4.97 \%$ and $96.80 \pm 3.52 \%$, respectively. The healing rate of each group at each time point was shown in Figure $3 \mathrm{~b}$. In a word, there was no significant difference in the therapeutic effect between KLCs and KCs, but the therapeutic effect of KLCs and KCs was much better than that of PBS and HFF-1. Compared with PBS and HFF-1, the wounds treated with KLCs and KCs shrank earlier. On the 9th day after injury, the wound healing rate in the KLCs group was even higher than that in the KCS group. The therapeutic effect of HFF-1 was also significantly higher than that of PBS. The results of HE staining showed that on the 3rd day囚the skin tissue structure of each group was severely abnormal, the epidermal layer fell off, and a large number of neutrophil infiltration was seen; the skin appendages were missing, no hair follicles, sebaceous glands and collagen fibers were seen, and a large number of cellulose-like structures were seen , A large number of pathological changes such as angiogenesis. On the 6th day, the skin tissue structure of the PBS group and HFF-1 group was abnormal, there was no obvious epidermal layer structure, and a large number of neutrophil infiltration was still seen; a large number of fibroblasts and inflammatory cells proliferated in some areas, a large number of angiogenesis, and no dermis layer was seen Sebaceous glands, hair follicles and collagen fibers can be seen with cellulose-like structures; but in the KLCs and KCs groups, the skin tissue structure is basically normal, the epidermal structure is intact, no cell degeneration is seen, most of the normal tissues can be seen under the epidermis, and a large number of skin appendages can be seen. Collagen fibers are arranged closely and regularly. On the 9th day, the skin tissue structure of the PBS group and HFF-1 group was slightly abnormal, the epidermal structure was relatively complete, some normal tissues were visible under the epidermis, a large number of skin appendages were visible, collagen fibers were arranged regularly, and a large number of inflammatory cells were seen in the tissue; KLCs The skin tissue structure of the KCs group and the KCs group is basically normal, the epidermal structure is intact, the collagen fibers of the dermis are arranged neatly, and the skin is closer to the physiological state. In short, the wound healing of the KLCS group and the KCS group is significantly better than that of the PBS group and HFF-1 Group (Figure 3c). To further confirm that KLCs are closely related to wound healing, CK5 antibody was used to perform immunohistochemical staining on the wound. The results showed that after transplantation of KLCs and KCs derived from HFF-1, a row of single cells remained between the epidermis and the dermis (Figure 3d), which played a role in connection during wound healing.In summary, the degree of re-epithelialization in the KLCs and KCs groups was significantly better than that of the HFF-1 and PBS groups, which could substantially promote wound healing.

\section{KLCs were not tumorigenic}

Safety is an issue that cannot be ignored in the future application of transformed KLCs cells in wound healing in clinical practice. Therefore, subcutaneous tumorigenesis assay was performed in nude mice. On the $40^{\text {th }}$ day after the injection of cells in each group, no obvious tumorigenesis was observed in the KCs and KLCs groups, but there was obvious growth of new organisms at the injection site in the Hepg2 group (control group). The tumor tissues of the KCs, KLCs and Hepg2 groups were removed and weighed 
(Figure 4a). HE staining of tumor tissue in the Hepg2 group revealed a large number of neutrophil infiltration, as shown by yellow arrows; local cells were loosely distributed, and the cytoplasm was vacuolated, as shown by red arrows; a large number of tumor cells in the tissue had increased atypia and obviously irregular nuclei, as shown by black arrows, while the KLCs and KCs groups showed the hair follicle structure and did not have the above-mentioned pathological features (Figure 4b).

Immunostaining of tumors with human CK5 antibody demonstrated that there was no carcinogenicity caused by KLCs in vivo (Figure 4d). These results indicate that KLCs do not have the potential to induce tumorigenesis and are safe for in vivo administration in the future.

\section{Identification of the mechanism underlying KLCs transformation}

To explore the potential mechanism underlying the transformation of HFF-1 cells into KLCs, we analyzed the mRNA profiles of HFF- 1 as well as HFF- 1 cultured for 25 days, KLCs and KCs by RNA-seq, generated a heat map of the mRNA, and clustered the four cell sample types by PCA analysis (Figure 5a). Candidate mRNAs for dynamic transformation from HFF-1 to HFF-1-KLCs and finally to KLCs were identified (Figure $5 b)$. The results showed that the mRNA expression profiles of KLCs and KCs were similar. We analyzed the significantly changed mRNAs based on their dynamic expression patterns and mapped 20 clusters. Among them, 6 color clusters showed that the enriched mRNA was detected, which was statistically significant. We found that the gene expressions of clusters 17 and 19 were up-regulated, while clusters 0 , 1,2 , and 7 were down-regulated. The p-values of up-regulated clusters 7 and down-regulated 17 were much lower than those of other clusters (Figure 5c). Therefore, clusters 7 and 17 were identified as the focus in our next research. KEGG enrichment analysis of the differential genes of clusters 7 and 19 respectively demonstrated that the transdifferentiation of HFF-1 into KLCs was closely related to the changes in the Wnt signaling pathway (Figure 5d).

\section{Activation of the Wnt/ $\beta$-catenin pathway promoted transformation of KLCs}

$\beta$-catenin, GSK-3 $\beta$, Axin1, APC and the TCF/LEF family of transcription factors are important members of the $\mathrm{Wnt} / \beta$-catenin signaling pathway. LEF1 can interact with nuclear $\beta$-catenin and act as the central transcription mediator of Wnt signaling [48], and Axin1 can form $\beta$-Catenin degradation complex with $A P C$ and GSK3 $\beta$ to inhibit $\beta$-Catenin aggregation in the cytoplasm or entering the nuclear transcription. Based on our previous RNA-seq results, we hypothesize that the transformation from HFF-1 to KLCs is caused by the activation of the Wnt/ $\beta$-catenin pathway. Western-blot results showed that with the progress of HFF-1 transformation, the levels of Axin 1 protein and $\beta$-catenin phosphorylated protein increased, resulting in a decrease in the total expression of $\beta$-catenin, and also significantly inhibiting the levels of APC and p-GSK- $3 \beta$ protein. 
From another perspective, the transdifferentiation of HFF-1 into KLCs can be considered as a process of mesenchymal epithelial transformation (MET), and ZEB1 is one of the most critical molecules in this process. The results revealed that $\beta$-catenin entered the nucleus and bound to the transcription factor LEF1 after its level increased, thereby increasing the level of LEF1, inhibiting the expression of ZEB1 and epithelial mesenchymal transformation (EMT), and promoting the occurrence of MET, that is, the transdifferentiation of HFF-1 into KLCs (Figure 6a, b). By immunofluorescence staining, $\beta$-catenin represented by the green fluorescence was distributed in the whole cell with high intensity in the HFF-1 group. However, in the KLCs group and KCs group cell species, the fluorescence intensity was reduced in the KLCs and KCs groups, and the fluorescence intensity in the nucleus was much lower than that in the cytoplasm, indicating that the expression of $\beta$-catenin decreased significantly, and the translocation of $\beta$ catenin in the nucleus was suppressed (Figure 6c).

Subsequently, to further explore the influence of the Wnt/ $\beta$-catenin signaling pathway on the transdifferentiation of HFF-1 to KLCs, HFF-1 was treated with the effective activator of the Wnt/ $\beta$-catenin

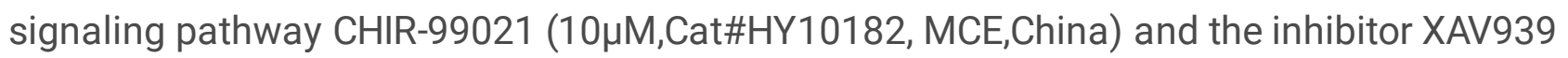
$(10 \mu \mathrm{M}, \mathrm{Cat} \# \mathrm{HY} 15147, \mathrm{MCE}, \mathrm{China})$. Surprisingly, HFF-1 treated with CHIR-99021 began to transform on the $10^{\text {th }}$ day, but this transformation did not occur in the unhandled, PBS and XAV939 groups (Figure 6d). The characteristics of transformed cells were further examined at the molecular protein level (Figure 6e, $\mathrm{f})$, and the results indicated that the activation of the $\mathrm{Wnt} / \beta$-catenin pathway played a key role in the transformation of KLCs.

\section{Discussion}

Our study revealed that fetal skin fibroblasts can spontaneously transdifferentiate into KLCs by accumulating over time with ordinary culture conditions. On the 25th day of HFF-1 culture, it was observed that the morphology of HFF-1 cells began to change, and the total transformation rate reached $98 \%$ on the 40th day. The transformed KLCs and KCs both highly expressed cytokeratins CK5, CK14, CK19, E-cadherin and integrin $\beta 1$, and did not express fibroblast-specific proteins Vimentin and a-SMA. From the perspective of cell proliferation and migration, transformed KLCs and KCs have similar performance. The activation of Wnt/ $\beta$-catenin signaling pathway played an important role in the transformation of HFF-1 to KLCs. The possible mechanism underlying this process was that with the activation of Wnt signaling, the stability of the degradation complex composed of $\beta$-catenin, axin 1 , GSK$3 \beta$, colorectal adenomatous polyp gene (APC) could be attenuated, thus preventing the degradation of $\beta$ catenin induced by phosphorylation and increasing its concentration in the cytoplasm. $\beta$-catenin then transferred into the nucleus and bound to lymphoid enhancer-binding factor-1 (LEF1) to inhibit the expression of the downstream target gene ZEB1 (a key factor in EMT) (Figure 7). The transformed KLCs were as effective as KCs in healing wounds, and were superior to PBS and fibroblasts. More importantly, with the treatment of KLCs and KCs, the immunohistochemical results showed hair follicle-like structures. Our future research will probe into the identification of these hair follicle-like structures and their sources. 
The phenomenon of spontaneous transdifferentiation of fetal fibroblasts reported in this study differs significantly from previous studies [30]. For example, it has been reported that dermal fibroblasts can transdifferentiate into KLCs phenotype in only 6 days, accounting for a transdifferentiation rate of about $50 \%$, which requires the combined use of inducers (p63 and KLF4) to enter dermal fibroblasts, and there are functional differences between KLCs and KCs after induction [31]. In the meantime, we found a report that presented similar findings with ours, namely, adult skin fibroblasts underwent similar transformations (from FBs to KLCs) on the 120th day during the routine culture in vitro, which was regulated by the PI3K-AKT pathway mediated by LINC00672 [32]. The difference in transformation time may be attributed to the stronger pluripotency and plasticity of fetal skin fibroblasts than that of adult skin fibroblasts. This further shows that human skin fibroblasts have strong pluripotency and plasticity when cultured in vitro,In addition, RNA-seq results show that the enrichment of PI3K-AKT signaling pathway ranks lower. We believe that the possible cause is the heterogeneity of fetal skin fibroblasts and adult skin fibroblasts. Many studies have been reported on the direct induction of skin fibroblasts into the cell types we need through reprogramming and other methods $[33,34]$. Therefore, our focus is on the potential of skin fibroblasts to spontaneously transdifferentiate into KLCs on wound healing and scar repair.

Skin wound healing occurs in a series of overlapped but different steps, and the most ideal outcome of this process is the remodeling of the epithelium and its appendages and the restoration of the epidermal barrier function. After skin injury, the wound healing is mainly manifested with the increase of KCs proliferation. This phenomenon occurs in the proliferation area around the wound, 0.5-1.5 mm away from the wound edge, but the proliferation of KCs was not observed at the wound edge [35-37]. Intravital microscopy revealed that during the wound healing process, both the basal layer and the super-basal layer migrated $[37,45]$ and the keratinocytes closer to the wound edge migrated faster, which both migrated and proliferated $0.5 \mathrm{~mm}$ away from the wound edge. $\mathrm{KCs}$ at the wound edge have a stronger migration ability than those in other parts. We hypothesized that the KCs at the wound edge may have undergone epithelial-mesenchymal transition (EMT), so that the epithelial KCs have acquired certain phenotypes of interstitial cells, which represents the ability to migrate. Re-epithelialization plays an important role in the entire wound healing process, involving the proliferation and migration of epidermal $\mathrm{KCs}$ around the wound. The dynamic mutual transformation of the proliferation and migration characteristics of KCs is similar to some aspects of EMT, so the healing process involves part of EMT [38]. Mesenchymal-epithelial transformation (MET) is the reverse process of EMT. In recent years, MET has been largely studied in the field of tumor. Metastatic tumor cells need continuous EMT-MET to colonize distant organs [39], and complete the distant tumor colonization by epithelial-mesenchymalepithelial transition. Few has explored the relationship between MET and wound healing. It is noteworthy that skin fibroblasts and epidermal stem cells colonized in the basal layer of the skin and hair follicles need to migrate to the wound to undergo terminal differentiation during the process of wound healing. We found that the transdifferentiation from fibroblasts into KLCs is a manifestation of MET, and proposed that this process is mainly regulated by the Wnt/ $\beta$-catenin-LEF1-ZEB1 signaling pathway(Figure7). It may also be jointly regulated by other unknown genes or signal pathways. It is 
therefore believed that wound healing is the result of the combined effect of the EMT of keratinocytes and the MET of fibroblasts, similar to the distant colonization of tumor cells.

Scars are a common consequence of skin injury, causing various adverse effects on patients, including physical deformities and mental illness [40,41].The excessive production of connective tissue (especially collagen) during wound healing may lead to scar formation $[42,43]$, which is closely associated with the synthesis of extracellular matrix (ECM) and the promotion of ECM remodeling by fibroblasts.Studies have shown that the skin damage of human fetuses in early pregnancy can be cured, forming normal skin tissues without leaving scars [44].The specific mechanism of scarless healing of fetal skin is not yet clear, but the in vitro transdifferentiation potential of fetal skin fibroblasts may represent a brand-new therapeutic option.

\section{Conclusions}

In this study, we found that fetal skin fibroblasts can spontaneously transdifferentiate into KLCs when cultured in ordinary DMEM nutrient solution for about 40 days, and this process may be regulated by the $W n t / \beta$-catenin signaling pathway. When the $W n t / \beta$-catenin pathway is activated, the fibroblast transformation time can be shortened from 40 days to 10 days. The transformed KLCs have similar biological characteristics as skin KCs, which promote wound healing, and have no obvious tumorigenicity. Therefore, the spontaneous transdifferentiation potential of HFF-1 can provide new ideas for extensive and scarless wound healing.

\section{Abbreviations}

HFF-1:Fetal skin fibroblasts;KCs: keratinocyte cells; FBs: fibroblasts; KLCs: keratinocyte-like cells; iPSCs: induced pluripotent stem cells; DMEM: Dulbecco`s Modified Eagle Media; PBS: phosphate-buffered saline; CK: Cytokeratin; a-SMA: alpha smooth muscle actin; RNA-seq: Transcriptome sequencing technology;EMT: epithelial-mesenchymal transition;MET: mesenchymal-epithelial transition;

\section{Declarations}

\section{Acknowledgements}

We are grateful to Guangzhou Genedenovo Biotechnology Co,. Ltd for assisting in sequencing and bioinformatics analysis.

\section{Authors' contributions}


H.Z., X.L., and C.S. conceived and designed the experiments. H.Z., K.Z., Z.L., J.Q.,and J.S. analyzed the data. H.Z., X.L.,K.Z.,Z.L.and J.S.performed the experiments. The manuscript was written by H.Z.,J,M.,Y.N.,and C.S. All authors read and approved the final manuscript.

\section{Funding}

This work was supported by the Major Program of National Key Research and Development Plan (2017YFC1103503), the Key Program of Beijing Natural Science Foundation (7171009) and the Key Program of Capital Clinical Application Research and Achievements Promotion (Z171100001017146).

\section{Ethics approval and consent to participate}

Human skin tissue sample were obtained from informed, consenting patients at the Fourth Medical Center of PLA General Hospital, This study was performed in compliance with the principles of the Helsinki Declaration and Guidelines for the Care and Use of Laboratory Animals of the Chinese Institute of Health. All procedures using animals were approved by the Animal Research Committee and Ethics Committee of General Hospital of PLA. We obtained the written informed consent from all the patients participated in this study.

\section{Availability of data and materials}

The datasets used and/or analyzed during the current study are available from the corresponding author on reasonable request.

\section{Consent for publication}

Not applicable

\section{Competing interests}

The authors declare that they have no competing interests.

\section{Author details}

${ }^{1}$ Jinzhou Medical University, No.82 Songpo Road, Guta District, Jinzhou 121001, Liaoning Province,China

${ }^{2}$ Department of Burn Surgery, Fourth Medical Center, PLA General Hospital,No 51 Fucheng Road, Haidian District, Beijing 100089,China 


\section{References}

1. Takahashi K, Yamanaka S. Induction of pluripotent stem cells from mouse embryonic and adult fibroblast cultures by defined factors. Cell. 2006 Aug 25;126(4):663-76.

2. Kocaefe YC, Israeli D, Ozguc M, Danos O, Garcia L. Myogenic program induction in mature fat tissue (with MyoD expression). Exp Cell Res. 2005 Aug 15;308(2):300-8.

3. leda M, Fu JD, Delgado-Olguin P, Vedantham V, Hayashi Y, Bruneau BG, Srivastava D. Direct reprogramming of fibroblasts into functional cardiomyocytes by defined factors. Cell. 2010 Aug 6;142(3):375-86.

4. Vierbuchen T, Ostermeier A, Pang ZP, Kokubu Y, Südhof TC, Wernig M. Direct conversion of fibroblasts to functional neurons by defined factors. Nature. 2010 Feb 25;463(7284):1035-41.

5. Ambasudhan R, Talantova M, Coleman R, Yuan X, Zhu S, Lipton SA, Ding S. Direct reprogramming of adult human fibroblasts to functional neurons under defined conditions. Cell Stem Cell. 2011 Aug 5;9(2):113-8.

6. Szabo E, Rampalli S, Risueño RM, Schnerch A, Mitchell R, Fiebig-Comyn A, et, al. Direct conversion of human fibroblasts to multilineage blood progenitors. Nature. 2010 Nov 25;468(7323):521-6.

7. Sekiya S, Suzuki A. Direct conversion of mouse fibroblasts to hepatocyte-like cells by defined factors. Nature. 2011 Jun 29;475(7356):390-3.

8. Zhang Y, Hu W, Ma K, Zhang C, Fu X. Reprogramming of Keratinocytes as Donor or Target Cells Holds Great Promise for Cell Therapy and Regenerative Medicine. Stem Cell Rev Rep. 2019 Oct;15(5):680-689.

9. Chen Y, Mistry DS, Sen GL. Highly rapid and efficient conversion of human fibroblasts to keratinocyte-like cells. J Invest Dermatol. 2014 Feb;134(2):335-344.

10. Pratsinis H, Mavrogonatou E, Kletsas D. Scarless wound healing: From development to senescence. Adv Drug Deliv Rev. 2019 Jun;146:325-343.

11. Prakoeswa CRS, Rindiastuti Y, Wirohadidjojo YW, Komaratih E, Nurwasis, Dinaryati A, Lestari NMI, Rantam FA. Resveratrol promotes secretion of wound healing related growth factors of mesenchymal stem cells originated from adult and fetal tissues. Artif Cells Nanomed Biotechnol. 2020 Dec;48(1):1160-1167.

12. Wang X, Shen C, Li Z, Xu S, Li D. Efficient isolation and high yield of epidermal cells from foreskin biopsies by dynamic trypsinization. Burns. 2018 Aug;44(5):1240-1250.

13. Zhang B, Sun P, Shen C, Liu X, Sun J, Li D,et, al. Role and mechanism of PI3K/AKT/FoxO1/PDX-1 signaling pathway in functional changes of pancreatic islets in rats after severe burns. Life Sci. 2020 Oct 1;258:118145.

14. Liu QW, Liu QY, Li JY, Wei L, Ren KK, et, al. Therapeutic efficiency of human amniotic epithelial stem cell-derived functional hepatocyte-like cells in mice with acute hepatic failure. Stem Cell Res Ther. 
2018 Nov 21;9(1):321.

15. Wu M, Ji S, Xiao S, Kong Z, Fang H, et, al. JAM-A promotes wound healing by enhancing both homing and secretory activities of mesenchymal stem cells. Clin Sci (Lond). 2015 Oct;129(7):57588.

16. Iyawaki S, Kawamura Y, Oiwa Y, Shimizu A, Hachiya T, et, al. Tumour resistance in induced pluripotent stem cells derived from naked mole-rats. Nat Commun. 2016 May 10;7:11471.

17. Zhang F, Wang L, Wang JJ, Luo PF, Wang XT, Xia ZF. The caspase-1 inhibitor AC-YVAD-CMK attenuates acute gastric injury in mice: involvement of silencing NLRP3 inflammasome activities. Sci Rep. 2016 Apr 7;6:24166.

18. Love MI, Huber W, Anders S. Moderated estimation of fold change and dispersion for RNA-seq data with DESeq2. Genome Biol. 2014;15(12):550.

19. Robinson M D. et al.edgeR: a Bioconductor package for differential expression analysis of digital gene expression data[J]. Bioinformatics.26:139-140 (2010).

20. Kanehisa M, Goto S. KEGG: kyoto encyclopedia of genes and genomes. Nucleic Acids Res. 2000 Jan 1;28(1):27-30.

21. Li M, Ma J, Gao Y, Dong M, Zheng Z, Li Y, Tan R, She Z, Yang L. Epithelial differentiation of human adipose-derived stem cells (hASCs) undergoing three-dimensional (3D) cultivation with collagen sponge scaffold (CSS) via an indirect co-culture strategy. Stem Cell Res Ther. 2020 Mar 31;11(1):141.

22. Taghiabadi E, Mohammadi P, Aghdami N, Falah N,et al. Treatment of Hypertrophic Scar in Human with Autologous Transplantation of Cultured Keratinocytes and Fibroblasts along with Fibrin Glue. Cell J. 2015 Spring;17(1):49-58.

23. Li X, Wang Y, Zhao Y, Yang H, Tong A, et al. Immunotherapy of tumor with vaccine based on basic fibroblast growth factor-activated fibroblasts. J Cancer Res Clin Oncol. 2014 Feb;140(2):271-80.

24. Celli A, Tu CL, Lee E, Bikle DD, Mauro TM. Decreased Calcium-Sensing Receptor Expression Controls Calcium Signaling and Cell-To-Cell Adhesion Defects in Aged Skin. J Invest Dermatol. 2021 Nov;141(11):2577-2586.

25. Luo Z, Dou J, Xie F, Lu J, Han Q, Zhou X, Kong J, Chen D, Liu A. miR-203a-3p promotes loureirin Ainduced hair follicle stem cells differentiation by targeting Smad1. Anat Rec (Hoboken). 2021 Mar;304(3):531-540.

26. Mini CA, Dreossi SAC, Abe FR, Maria-Engler SS, Oliveira DP. Immortalized keratinocytes cells generates an effective model of Epidermal Human Equivalent for irritation and corrosion tests. Toxicol In Vitro. 2021 Mar;71:105069.

27. Zhang C, Fu X, Chen P, Bao X, Li F, Sun X, Lei Y, Cai S, Sun T, Sheng Z. Dedifferentiation derived cells exhibit phenotypic and functional characteristics of epidermal stem cells. J Cell Mol Med. 2010 May;14(5):1135-45.

28. Hayward CJ, Fradette J, Morissette Martin P, Guignard R, Germain L, Auger FA. Using human umbilical cord cells for tissue engineering: a comparison with skin cells. Differentiation. 2014 MarApr;87(3-4):172-81. 
29. Norman MU, Chow Z, Snelgrove SL, Prakongtham P, Hickey MJ. Dynamic Regulation of the Molecular Mechanisms of Regulatory T Cell Migration in Inflamed Skin. Front Immunol. 2021 May 10;12:655499.

30. Ward C, Volpe G, Cauchy P, Ptasinska A, Almaghrabi R, et al.Fine-Tuning Mybl2 Is Required for Proper Mesenchymal-to-Epithelial Transition during Somatic Reprogramming. Cell Rep. 2018 Aug 7;24(6):1496-1511.e8.

31. Chen Y, Mistry DS, Sen GL. Highly rapid and efficient conversion of human fibroblasts to keratinocyte-like cells. J Invest Dermatol. 2014 Feb;134(2):335-344.

32. Zhang F, Zhang D, Cheng K, Zhou Z, Liu S, Chen L, Hu Y, Mao C, Liu S. Spontaneous evolution of human skin fibroblasts into wound-healing keratinocyte-like cells. Theranostics. $2019 \mathrm{Jul}$ 9;9(18):5200-5213.

33. Koren S, Reavie L, Couto JP, De Silva D, Stadler MB, et al. PIK3CA(H1047R) induces multipotency and multi-lineage mammary tumours. Nature. 2015 Sep 3;525(7567):114-8.

34. Liu Y, Giannopoulou EG, Wen D, Falciatori I, Elemento O,et al. Epigenetic profiles signify cell fate plasticity in unipotent spermatogonial stem and progenitor cells. Nat Commun. 2016 Apr 27;7:11275.

35. Aragona M, Dekoninck S, Rulands S, Lenglez S, Mascré G,et al. Defining stem cell dynamics and migration during wound healing in mouse skin epidermis. Nat Commun. 2017 Mar 1;8:14684.

36. Park S, Gonzalez DG, Guirao B, Boucher JD,et al. Tissue-scale coordination of cellular behaviour promotes epidermal wound repair in live mice. Nat Cell Biol. 2017 Mar 1;19(2):155-163.

37. Rodrigues M, Kosaric N, Bonham CA, Gurtner GC. Wound Healing: A Cellular Perspective. Physiol Rev. 2019 Jan 1;99(1):665-706.

38. Haensel D, Dai X. Epithelial-to-mesenchymal transition in cutaneous wound healing: Where we are and where we are heading. Dev Dyn. 2018 Mar;247(3):473-480.

39. Yang J, Weinberg RA. Epithelial-mesenchymal transition: at the crossroads of development and tumor metastasis. Dev Cell. 2008 Jun;14(6):818-29.

40. Monavarian M, Kader S, Moeinzadeh S, Jabbari E. Regenerative Scar-Free Skin Wound Healing. Tissue Eng Part B Rev. 2019 Aug;25(4):294-311.

41. Lipman K, Wang M, Berthiaume E, Holloway J, Da Lio A, Ting K, Soo C, Zheng Z. Evaluating Current Scar Assessment Methods. Ann Plast Surg. 2020 Feb;84(2):222-231.

42. Park JW, Hwang SR, Yoon IS. Advanced Growth Factor Delivery Systems in Wound Management and Skin Regeneration. Molecules. 2017 Jul 27;22(8):1259.

43. Harsha, L. et al. Role of collagen in wound healing. Drug Invent. Today 2020, 13, 55-57.

44. Li M, Zhao Y, Hao H, Han W, Fu X. Theoretical and practical aspects of using fetal fibroblasts for skin regeneration. Ageing Res Rev. 2017 Jul;36:32-41.

45. Kobayashi W, Ozawa M. The transcription factor LEF-1 induces an epithelial-mesenchymal transition in MDCK cells independent of $\beta$-catenin. Biochem Biophys Res Commun. 2013 Dec 6;442(1-2):1338. 


\section{Figures}

a

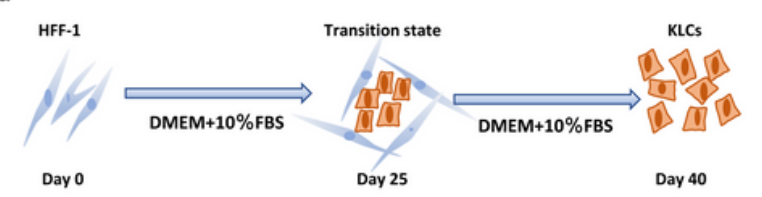

b
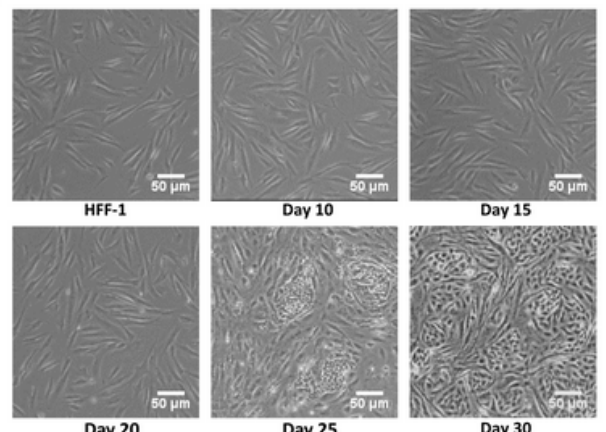

Day 25
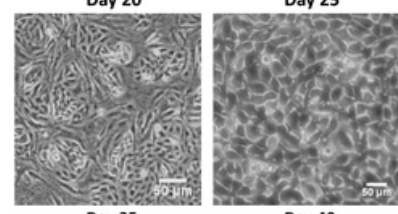

Day 40

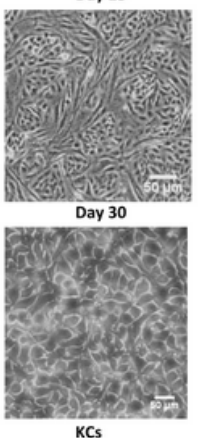

KCs
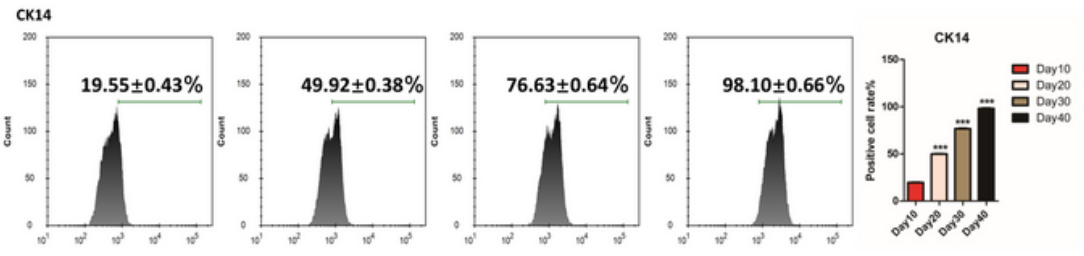

Vimentin
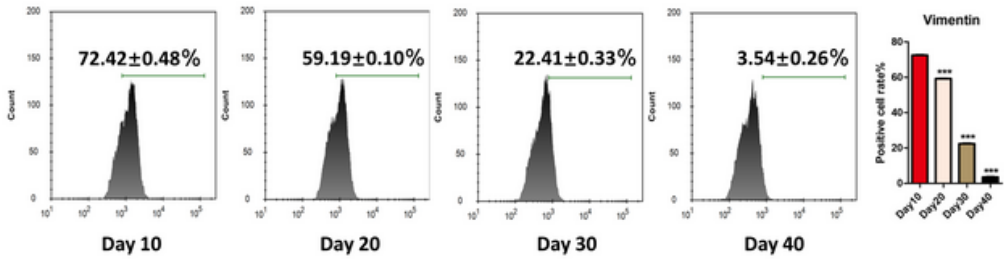

Dog 10
Doar20
Dar30
Days

d
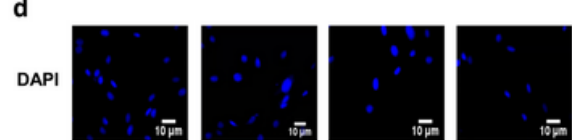

DAPI
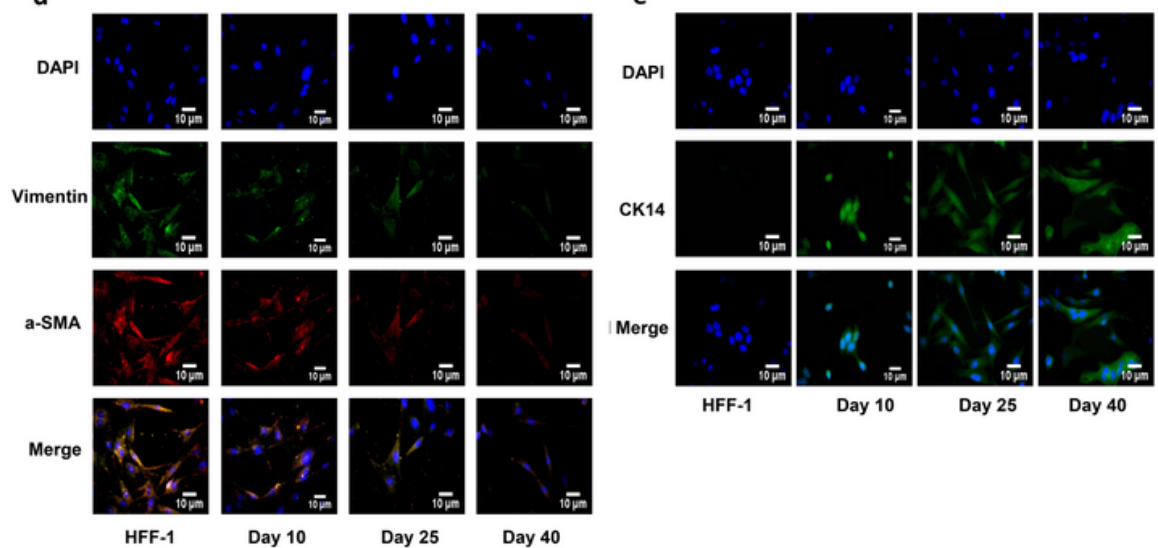

Figure 1

Fetal skin fibroblasts cultured in vitro have the potential to spontaneously transdifferentiate into KLCS. a Schematic diagram of transdifferentiation from HFF-1 to KLCs. b Brightfield image of spontaneous transdifferentiation from HFF-1 to KLCs over time. c Flow cytometry analysis of KCs labeled protein (CK14) and HFF-1 labeled protein (Vimentin) during HFF-1 transformation. d Immunofluorescence labeling analysis of the expression of HFF-1 specific proteins Vimentin and a-SMA in the process of transdifferentiation. e Immunofluorescence labeling analysis of the expression of KCs specific protein CK14. $(* \star * P<0.001, n=3)$ 
a

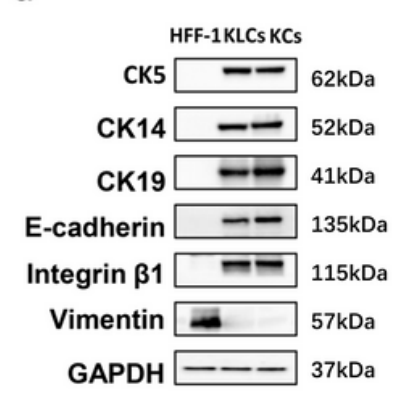

b

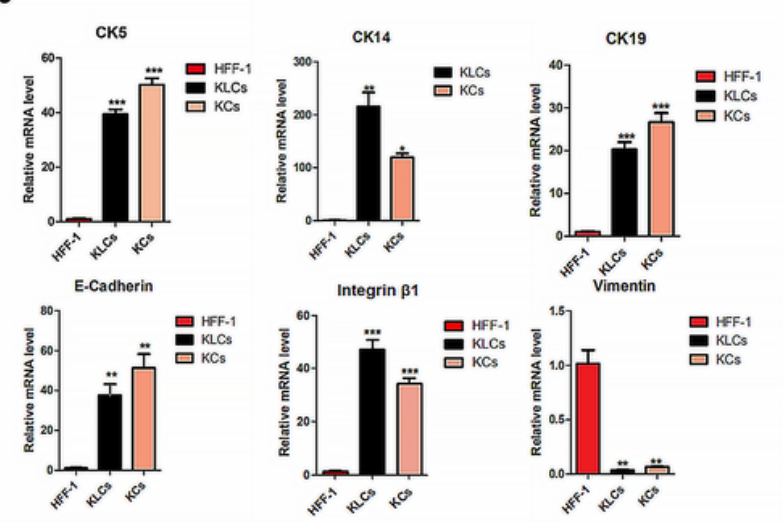

C

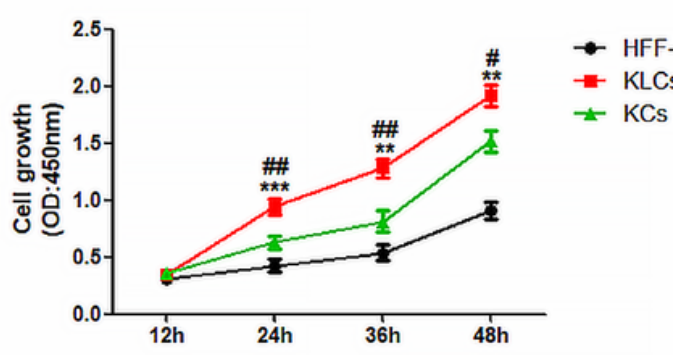

d

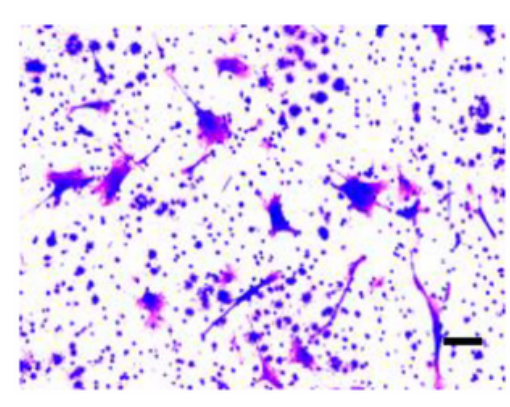

HFF-1

e
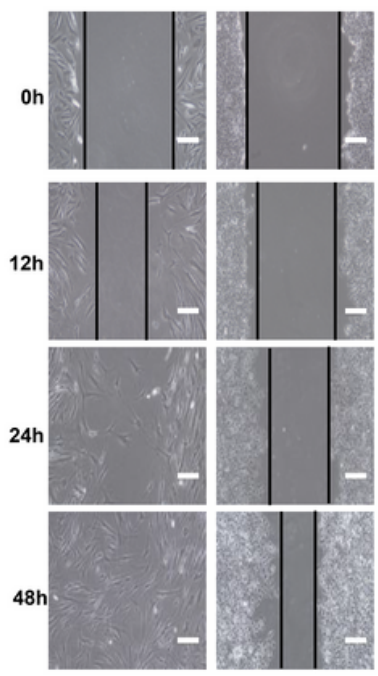

HFF-1
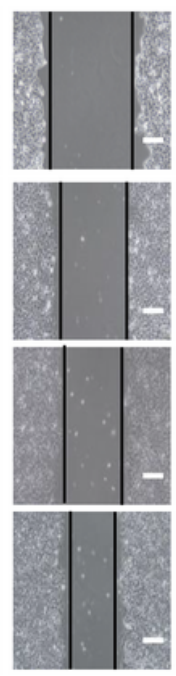

KCs
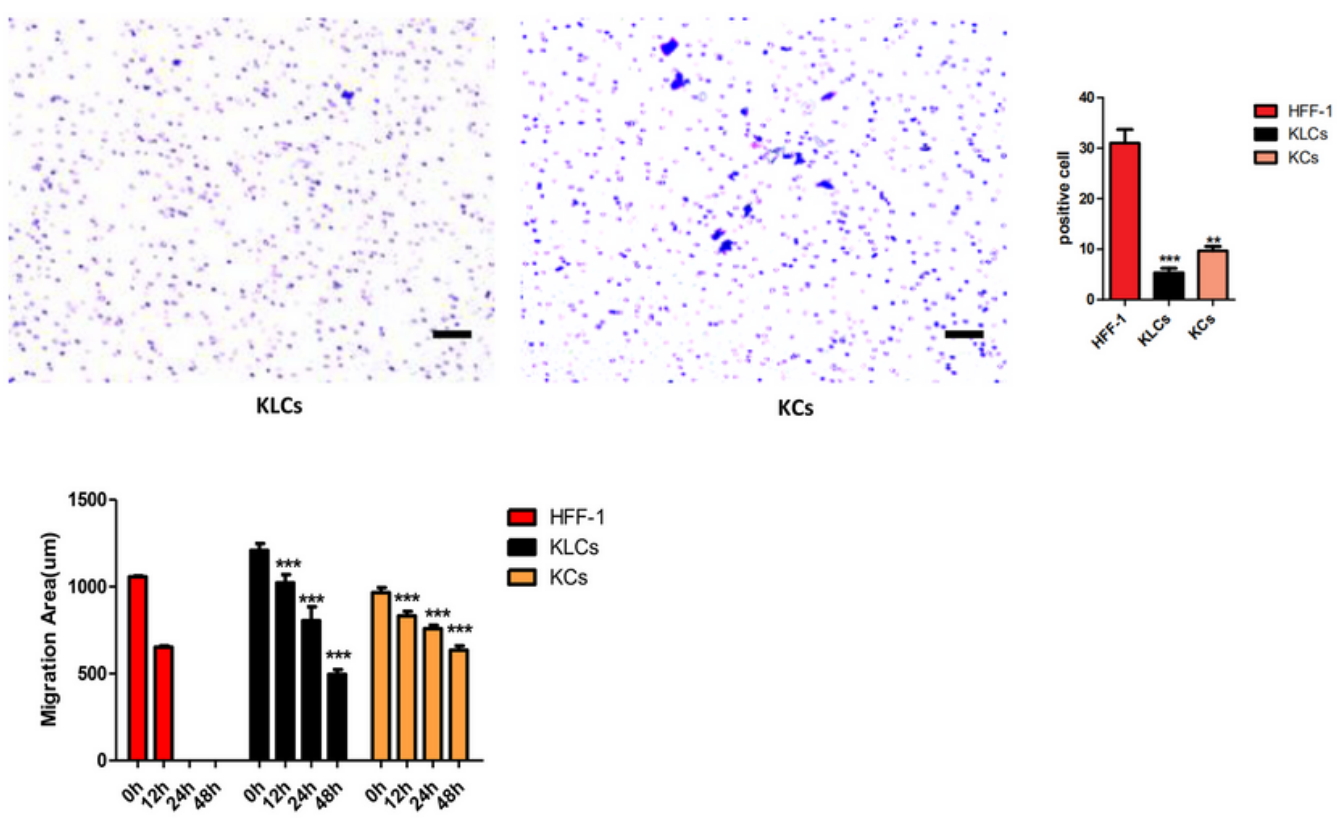

Figure 2

Features of KLCs. a Western blot showed that KLCs highly expressed CK5, CK14, CK19, E-cadherin and Integrin $\beta 1$, but did not express Vimentin $(n=3)$.b Quantitative reverse transcription polymerase chain reaction was used to detect the gene expression of CK5, CK14, CK19, E-cadherin,Integrin $\beta 1$ and vimentin. $\left({ }^{*} P<0.05,{ }^{*} P<0.01,{ }^{*} * P<0.001, n=3\right) . c$ CCK8 assay showed the proliferative ability of HFF-1, KLCs and $\mathrm{KCs}\left({ }^{\star *} \mathrm{P}<0.01\right.$, ${ }^{\star \star \star} \mathrm{P}<0.001$, HFF-1 group vs $\mathrm{KC}$ group, $\# \mathrm{P}<0.05$, \#\#P<0.01, HFF-1 group vs KLCs group, $n=3)$. $d$ Transwell test was used to analyze cell migration, and the number of positive cells was counted under the microscope. Scar bar, $10 \mu \mathrm{m}\left({ }^{\star *} \mathrm{P}<0.01,{ }^{\star *} \mathrm{P}<0.001, \mathrm{n}=3\right)$. e Wound scratch assay was 
employed to observe the migration of HFF-1, KLCs and KCs, and the wound width of HFF-1, KLCs and KCs at $0 \mathrm{~h}, 12 \mathrm{~h}, 24 \mathrm{~h}$ and $48 \mathrm{~h}$ were calculated. Scar bar, $10 \mu \mathrm{m}(* \star * \mathrm{P}<0.001, \mathrm{n}=3)$.

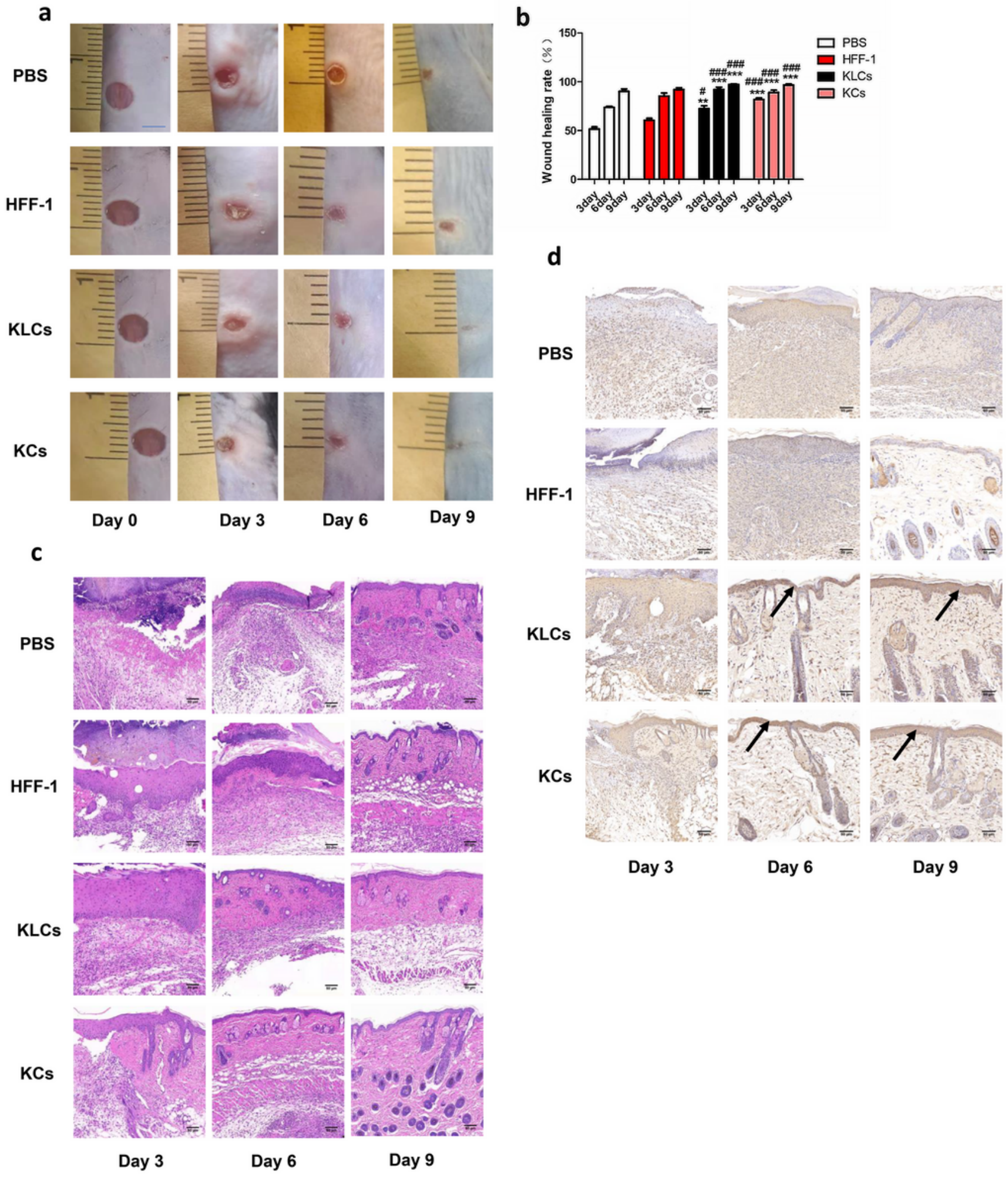

Figure 3

KLCs promoted wound healing. a Representative images showing wound healing on days 0, 3, 6 and 9 in PBS, HFF-1, KLCs and KCs groups $(n=5)$. b Wound healing rates on days 0 and 39 in PBS, HFF-1, KLCs and KCs groups ( ${ }^{* *} P<0.01,{ }^{*} * * P 0.001$, with significant difference compared with the PBS group; $\# p<0.05$, 
\#\#\#p<0.001, with significant difference compared with the HFF-1 group, $n=5)$. c HE staining of skin tissues in PBS, HFF-1, KLCs and KCs groups on 0, 3, 6, 9 days $\varangle n=3 \rrbracket$. $d$ Immunohistochemical staining showed that the mice wounds treated with KLCs contained CK5-positive cells, which is indicated by the black arrow $(n=3)$.

a

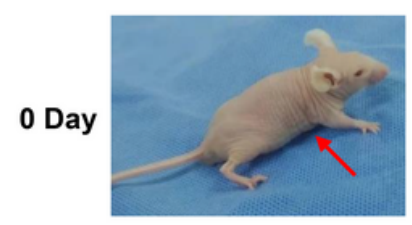

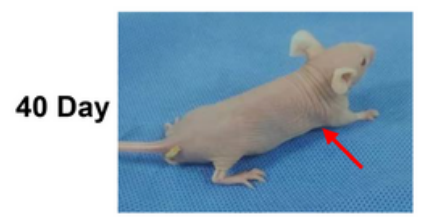

$\mathrm{KCs}$
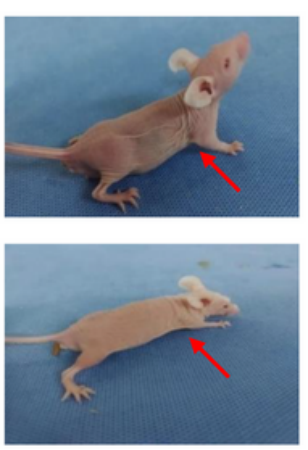

KLCs
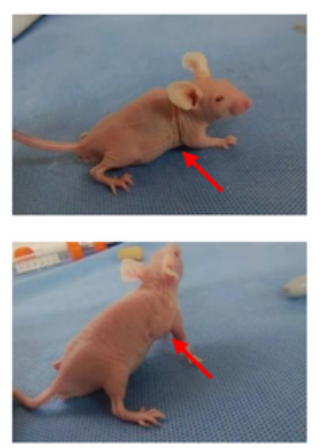

Hepg2

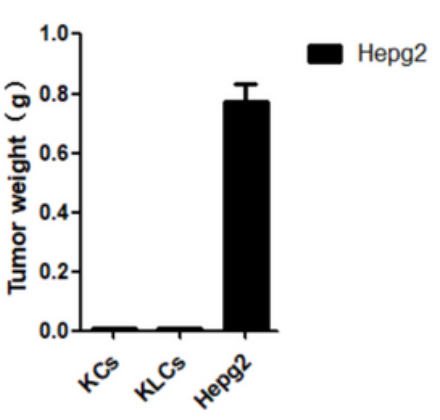

b

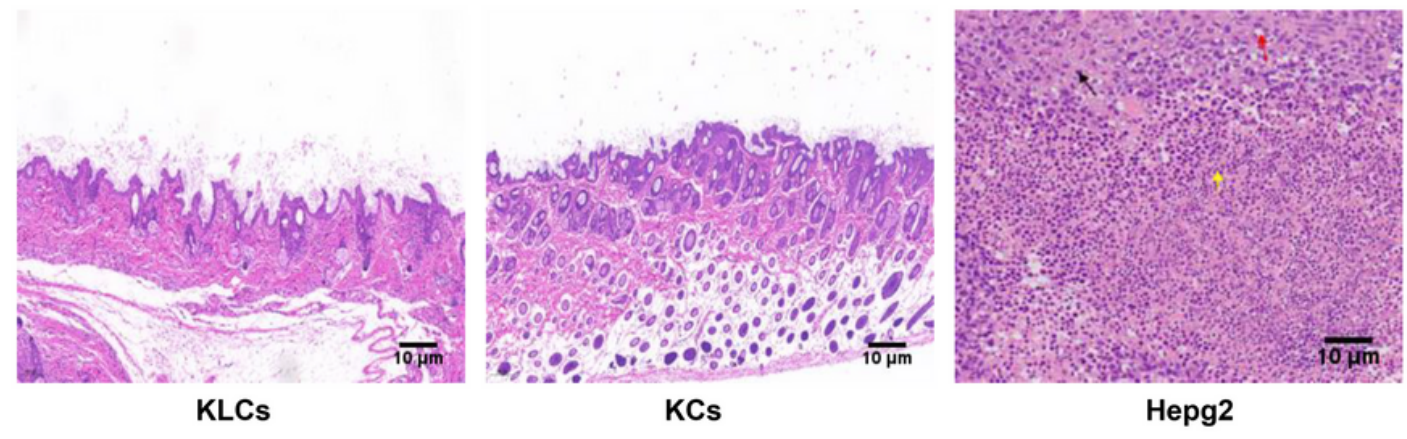

C

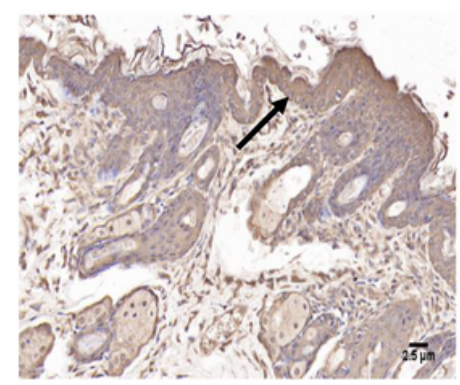

KCs

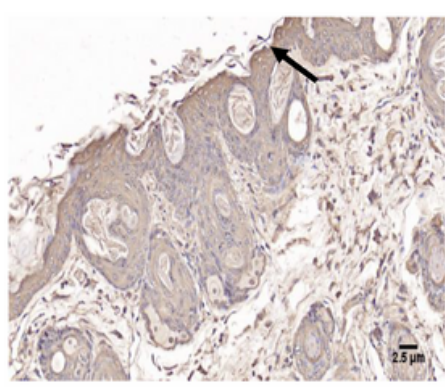

KLCs

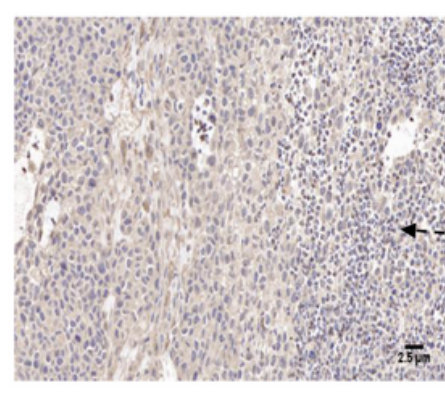

Hepg2

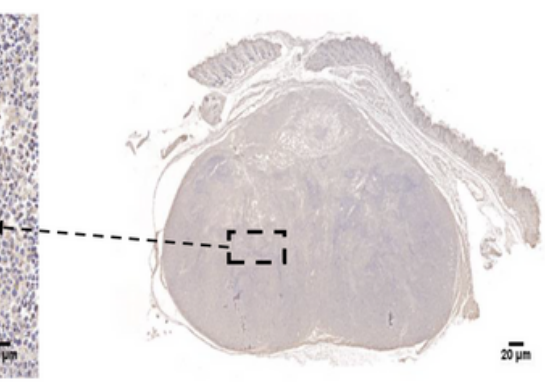

Hepg2

\section{Figure 4}

KLCs were not tumorigenic. a The tumorigenicity test of KLCs, KCs and Hepg2 cells in nude mice (the area indicated by the red arrow on day 0 is the cell injection site, and the area marked by the red arrow on day 40 is subcutaneous tumor on day 40 after cell injection, ${ }^{* *} P<0.001, n=5$ ). b HE staining of tumors in the KLCs, KCs and Hepg2 groups. KLCs and KCs group showed normal skin tissue, while there was a large number of infiltrated neutrophils in the Hepg2 group was observed, as shown by the yellow arrow; the local cells were loosely arranged, and the cytoplasm was vacuolated, as shown by the red arrow; a large 
number of tumor cells in the tissue increased atypia, and the nucleus was obviously irregular, as indicated by the black arrow $(n=5)$. c Immunohistochemical staining revealed CK5-positive cells in tumor tissues in the KLCs, KCs and Hepg2 groups ( $n=5)$.
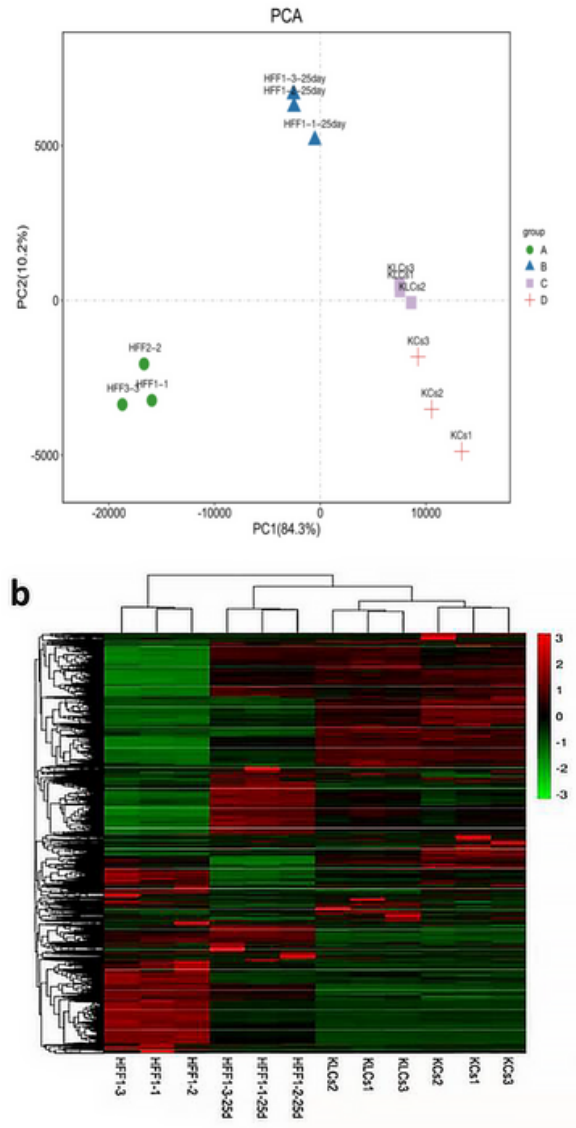

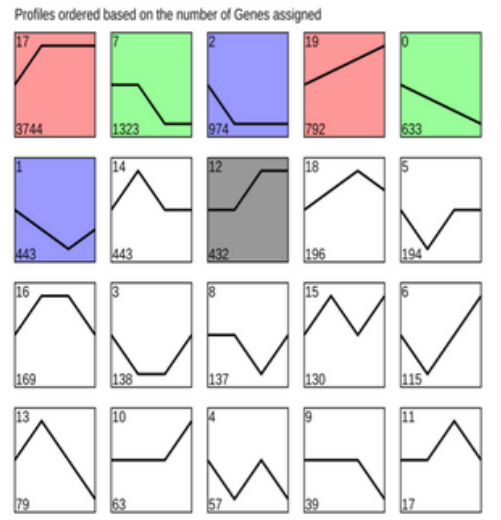

d
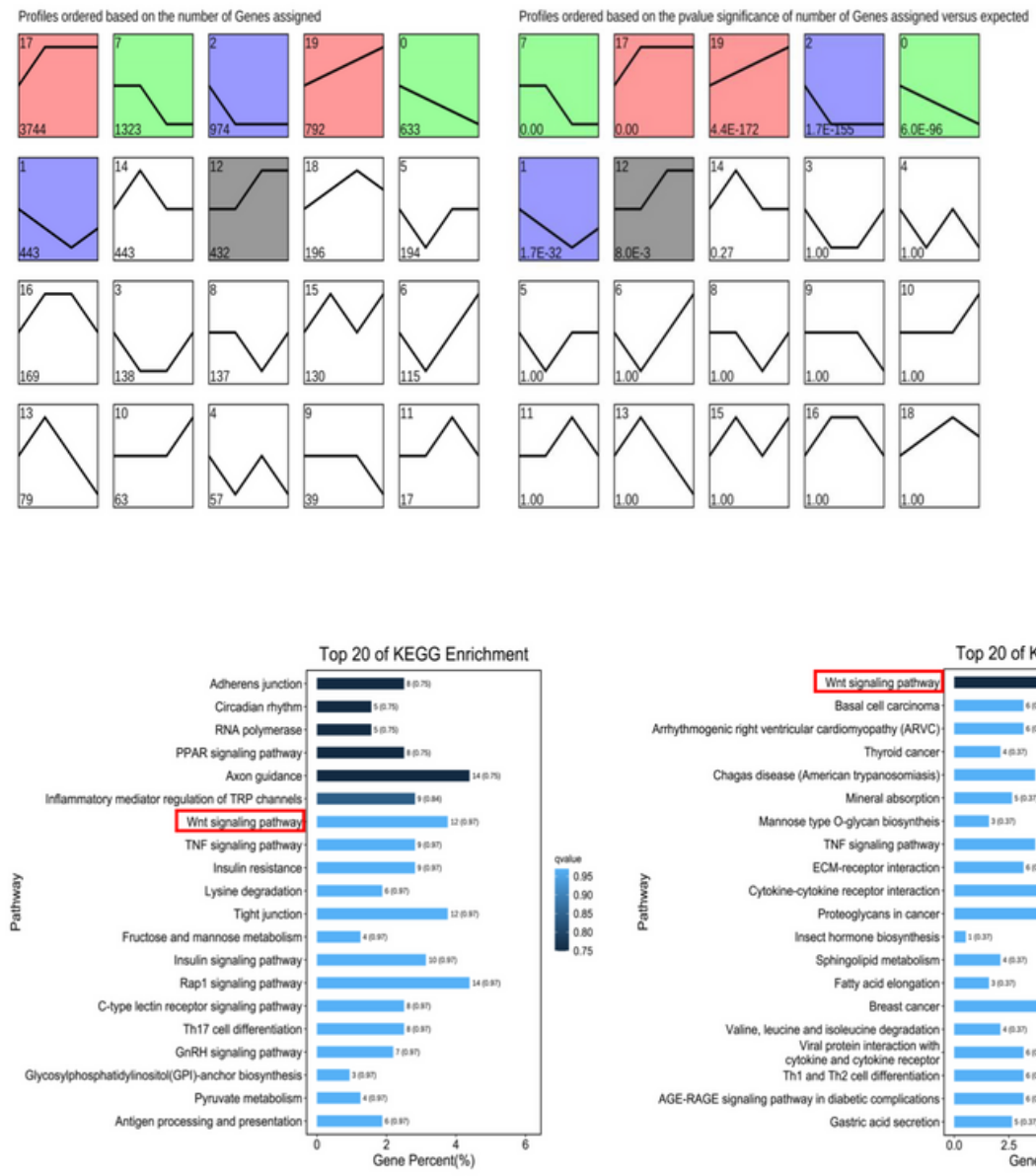

Figure 5

Identification of the mechanism of KLCs transformation. a PCA was used to analyze the composition of each group of samples. The more similar the sample composition is, the closer the distance reflected in the PCA chart is. The samples from different effective treatments often aggregate. b Heat maps of differentially expressed genes determined by RNA microarray analysis of HFF-1, HFF-1 (day 25), KLCs and KCs. c According to the gene expression patterns of each group of cell stages, 20 clusters were divided from the number and $\mathrm{P}$ value of differential genes, and the number of clusters is shown in the upper left corner. $d$ The pathways with significant enrichment of differential genes were obtained and the most important biochemical metabolic pathways and signal transduction pathways involved in differential genes were determined through KEGG analysis on No. 7 and No. 17 clusters. 

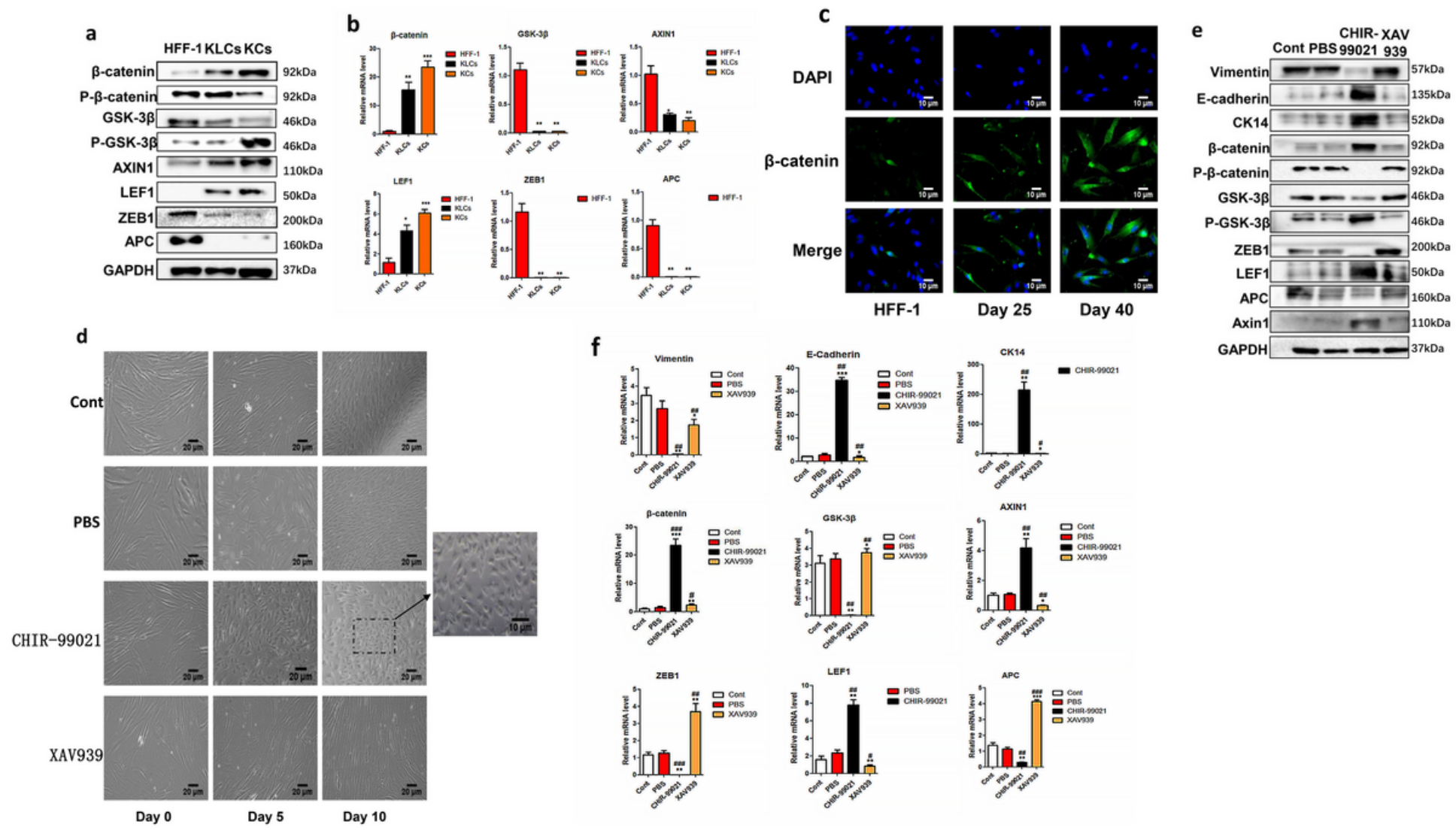

f
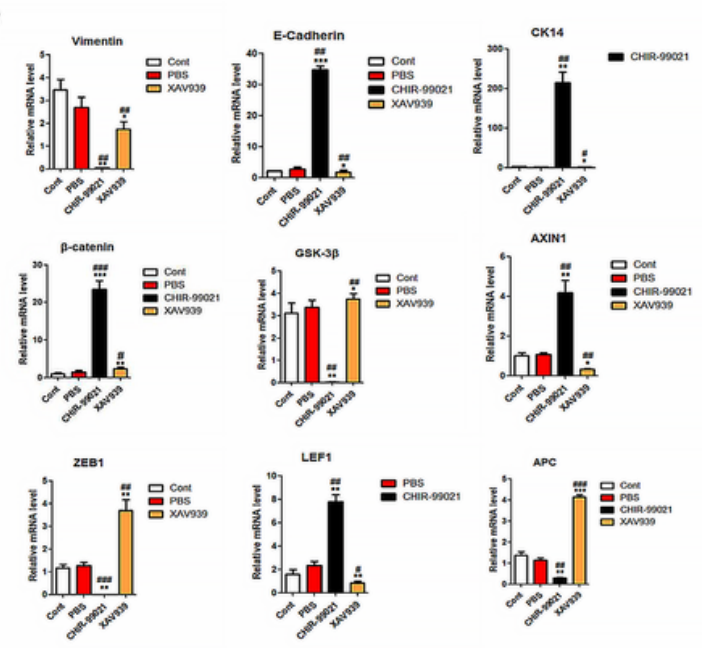

\section{Figure 6}

Wnt/ $\beta$-catenin pathway activation promoted KLCs transformation. a The expression of Wnt pathwayrelated protein in HFF-1, KLCs and KCs group cells $(n=3)$. b Quantitative reverse transcription polymerase chain reaction was used to detect the gene expression of Wnt pathway-related molecules ( ${ }^{*} \mathrm{p} \otimes 0.05$, ${ }^{*} \mathrm{p} \rrbracket$ $0.01, * \star * p \otimes 0.001, n=3)$. c Immunofluorescence analysis of $\beta$-catenin protein in HFF-1, HFF-1 (day 25) and HFF-1 (day 40) cells. $\beta$-catenin (green), Dapi (blue) staining and combined images showing the expression of $\beta$-catenin $(n=3)$. d HFF-1 was treated respectively with PBS, CHIR-99021 and XAV939, and the dynamic process of cell transdifferentiation in each group was recorded. e The expression of Vimentin, E-cadherin, CK14 and Wnt pathway related proteins after 10 days of treatment with PBS, CHIR99021 and XAV939. $f$ Quantitative reverse transcription polymerase chain reaction was used to detect the gene expression of E-cadherin, vimentin, CK14 and Wnt pathway related molecules after 10 days of treatment with PBS, CHIR-99021 and XAV939 ${ }^{*} p<0.05$, ${ }^{* \star} p<0.01,{ }^{* \star *} p<0.001$, with significant difference compared with the Cont group; $\# p<0.05$, \#\#P<0.01, \#\#\#p<0.001; with significant difference compared with the PBS group, $n=3$ ). 


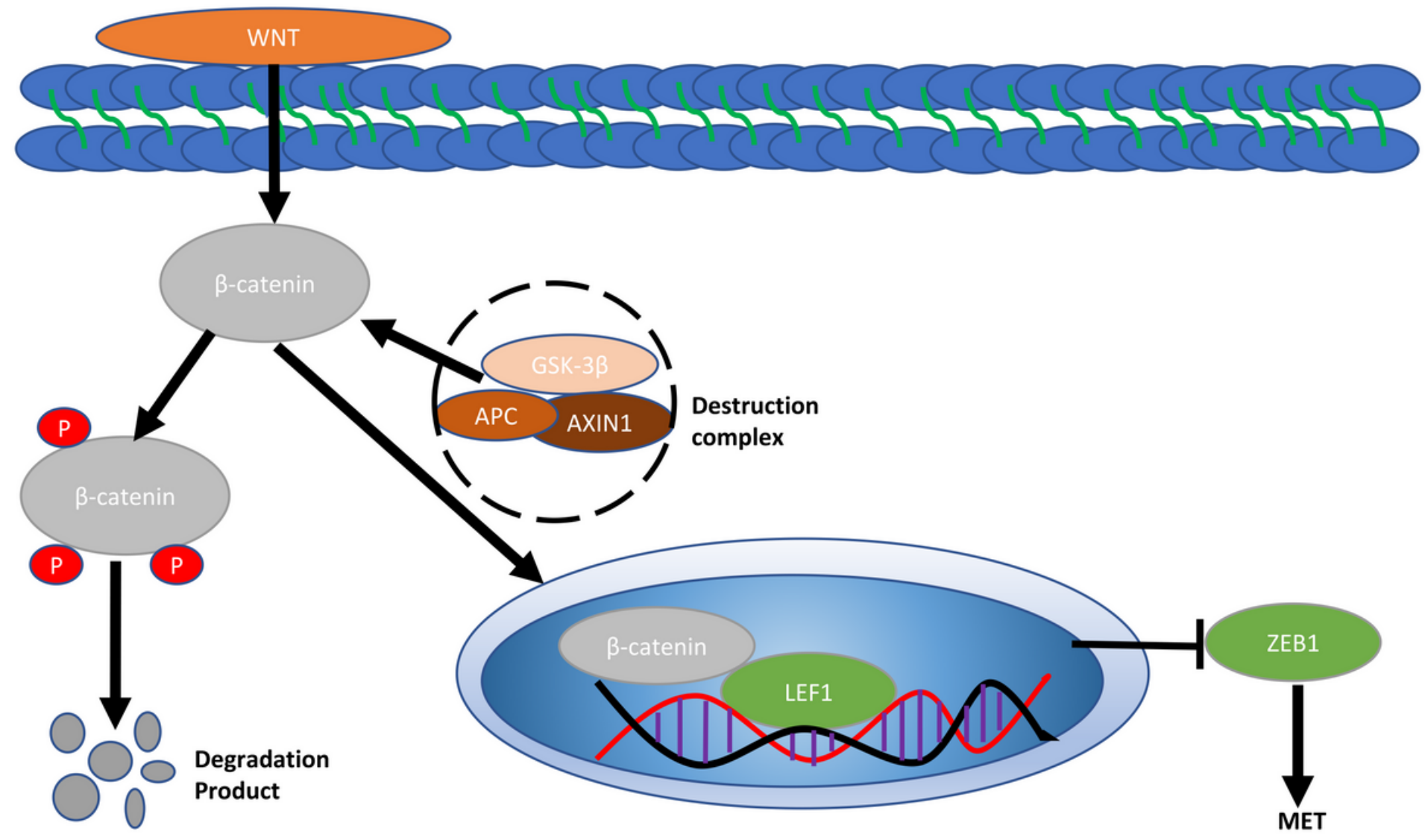

Figure 7

Molecular mechanism of HFF-1 transdifferentiating into KLCs. 